\title{
Parasitological Society of Southern Africa
}

The following are abstracts of papers and posters presented at the 32nd Annual Scientific Meeting of the Parasitological Society of Southern Africa (PARSA) in association with the South African Society of Travel Medicine (SASTM), 7-10 October 2003, at the Farm Inn, Pretoria.

\section{Abstracts of Papers}

\section{Malaria case management in South Africa: the national perspective}

\section{Blumberg}

National Institute for Communicable Diseases, PO Box X4, Sandringham, 2131 South Africa; lucilleb@nicd.ac.za

In South Africa, malaria case management is an important component of the malaria control programme and focuses on the prevention of malaria-related mortality and morbidity, rapid elimination of parasitaemia to minimise transmission, and limiting the emergence and spread of drug resistance. The majority of malaria cases $(>95 \%)$ are due to infection with Plasmodium falciparum. South Africans of all ages resident in the malaria transmission areas in the country, as well as those who acquire malaria post travel, would be considered nonimmune and therefore at risk for severe disease. Drug treatment policy is made at national level, and implemented at the operational level (provincial). Drug selection for case management is based on parasite resistance in the specific area where transmission occurs, drug safety, cost, suitability of regimen for outpatient treatment and severity of disease. Combination therapy using an artemisininderivative has been adopted to minimise the further development of drug resistance. Treatment is administered in the public sector through primary health clinics and cases of severe malaria referred to a hospital. Treatment is based on a confirmed diagnosis, using either a blood smear or a rapid antigen test. High-level chloroquine resistance is widespread, and sulfadoxine pyrimethamine resistance has emerged in KwaZulu-Natal, necessitating ongoing changes in treatment policy. Different regimens are recommended in the 3 provinces where malaria is transmitted because of differences in parasite resistance patterns. Currently, 1st-line treatment for uncomplicated malaria is as follows: KwaZulu-Natal, artemether lumefantrine; Mpumalanga, artesunate plus sulfadoxine pyrimethamine, and Limpopo, sulfadoxine pyrimethamine. In vitro and in vivo programmes are in place to detect further development of drug resistance and allow timely change in drug policy.

\section{Traditional methods versus geometric morphometric analysis in determining relatedness in 3 Amblyomma species}

\section{F C Clarke ${ }^{\mathrm{a}}$ and E Pretorius ${ }^{\mathrm{b}}$}

${ }^{a}$ Department of Biology, Medical University of Southern Africa, PO Box 139, MEDUNSA, 0204 South Africa; and ${ }^{b}$ Department of Anatomy, University of Pretoria, PO Box 2034,Pretoria, 0001 South Africa; clark@medunsa.ac.za

There are at present 10 species in the genus Amblyomma that are capable of transmitting Cowdria ruminantium in Africa. Amblyomma hebraeum, $A$. variegatum and $A$. gemma are probably the main vectors of C. ruminantium in sub-Saharan Africa. The 3 species are very similar in adult appearance, they exhibit the same host prevalence as adults, they occur in the same habitat types and their distributions overlap. A geometric morphometric analysis of the male and female body shape of the 3 species was performed. This analysis compared the body shape of the ticks with each other by collecting 17 landmarks from the ventral area of males and females and analysing this with geometric morphometrics. Thin-plate-spline analyses were performed on the consensus shape of the males and females, which indicated that the body shape of $A$. gemma and $A$. variegatum females could not be distinguished from each other. The male body shape of these 2 species is also very similar. The relative warp and the thin-plate-spline analyses separate A. hebraeum from the other 2 species.

In 6 individual experiments, a rabbit was infested with males of 1 of the 3 Amblyomma species and females of another to establish the crossbreeding potential of the different combinations of the 3 species. Engorged females were collected from all 6 rabbits and oviposition was recorded in all cases. Larvae hatched, although sometimes less than ten from the eggs in 5 of the 6 cases. Where possible, a rabbit was infested with flat larvae from 1 of the successful crosses. The only crosses resulting in male and female flat adults were $A$. hebraeum males $\times A$. gemma females and $A$. gemma males $\times A$. hebraeum females. The cross $A$. variegatum males $\times A$. hebraeum females resulted in flat females only. The results from the conventional method determining the potential to crossbreed do not correspond with that of geometric morphometrics.

\section{Equine anthelmintic resistance in the Western Cape Province}

\section{F H Dreyer}

Western Cape Provincial Veterinary Laboratory, Private Bag X5020, Stellenbosch, 7599 South Africa; francoisd@wcape.agric.za

A number of papers have been published in recent years on the topic of anthelmintic resistance of ovine nematodes in South Africa. Such information is, however, lacking for the local equine industry, with only 2 studies having been published by the beginning of 2000 . At the beginning of 2000 an investigation was done concerning anthelmintic resistance on 10 equine stud farms in the Western Cape Province and linked to this, data regarding certain managerial practices that could have had an influence on the resistance status of the farms was also collected. Findings from the study showed that resistance against oxibendazole occurred on all of the farms, with efficacies ranging from $0-88 \%$. Moxidectin had efficacies of between 99 and $100 \%$ and ivermectin had $100 \%$ efficacy on all the study farms. Other findings showed high drenching frequencies on most farms for all age groups. The study also considered factors such as faecal removal from pastures, number of alternations between drug groups on an annual basis, methods used for dosage calculation and stocking rates. The study identified certain areas that could possibly contribute to the development of resistance and aspects in need of further investigation.

\section{Micromorphology of the giant vulture louse Laemobothrion vulturis}

\section{E D Green ${ }^{\mathrm{a}}, \mathrm{M}$ L Turner $^{\mathrm{b}}$ and F C Clarke}

${ }^{a}$ Department of Anatomy, ${ }^{b}$ Electron Microscope Unit, and ${ }^{c}$ Department of Biology, Medical University of Southern Africa, PO Box 232, MEDUNSA, 0204 South Africa; edward@medunsa.ac.za

Laemobothrion vulturis is the largest known bird louse infesting several species of vultures and large eagles, many of which are endangered. This study was aimed at investigating the micromorphology of L. vulturis by scanning electron microscopy (SEM) to obtain a greater understanding of how these lice attach to, orientate and feed on their hosts. Lice were collected from disabled Cape griffons kept at the De Wildt Cheetah and Wildlife Centre, Pretoria. The lice were fixed in $70 \%$ ethanol, routinely prepared for SEM and viewed in a Leica Stereoscan 420 scanning electron microscope at 5-10 kV. The studies revealed a long louse that is dorsoventrally flattened to fit into the grooves between the feather barbs. It has an anteriorly elongated head with the mouthparts and large hypopharynx anteroventrally situated. The mandibles are specialised to grasp and shear off pieces of the feather barbule. The anterior maxillary palpi has 4 segments and 14 short, peglike, grooved terminal sensilla while the medial labial palpi has 5 terminal, grooved sensillae. The antennae have 4 segments, the terminal segment being pedunculate and bearing the sensory sensilla. Posterior to the antennal groove are the eyes, each consisting of a pair of separate lens-like ommatidia. Ventral to the eyes is a unique organ of unknown function, which consists of five rows of comb-like setae adjacent to an area covered by small scales. The prothorax is well 
developed while the meso-metathorax resembles the abdominal segments in shape. Each of the legs terminates in 2 horn-shaped tarsal claws without the normal opposing pretarsal setae found in other feather-infesting lice. The femur, however, has a medial groove ventrally, into which the tibia fits, enabling the feather barbules to be held firmly. The ventromedial area of femur III as well as the lateral areas of sternites V-VI are covered with microtrichia arranged as short comb-like structures, which are characteristic of Laemobothrion species. The round abdominal spiracles are carried dorsally on the tergites of the abdominal segments III-VIII while segments I-II only have small stigmatal scars. A pair of large comma-shaped thoracic spiracles is present on the lateral surfaces of the prothorax. The lumen is lined by parallel lamellae to form a fine filter mechanism. This study elucidated a number of micromorphological structures that may be of taxonomic importance in future studies of Laemobothrion lice.

\section{Functional specialisations of the louse Docophoroides brevis from the wandering albatross}

\section{E D Green ${ }^{\mathrm{a}}$ and M L Turner}

${ }^{a}$ Department of Anatomy and ${ }^{b}$ Electron Microscope Unit, PO Box 232, Medical University of Southern Africa, MEDUNSA, 0204 South Africa;

edward@medunsa.ac.za

Docophoroides brevis is a large feather louse that must remain attached to its host, the wandering albatross, even when submerged. This study investigated the micromorphology of $D$. brevis by scanning electron microscopy (SEM) to obtain a greater understanding of how these lice attach to, orientate and feed on their hosts. Lice were collected from nesting wandering albatrosses on Marion Island. The lice were fixed in $70 \%$ ethanol, routinely prepared for SEM and viewed in a Leica Stereoscan 420 scanning electron microscope at 5 to $10 \mathrm{kV}$. The studies revealed a robust louse that is dorsoventrally flattened with a large, flattened, shovel-shaped head to fit in between the feather barbules. The prominent preantennal processes and hooked post-antennal processes and the mandibles attach the head to the feather barbules. The mandibles are anteriorly notched and medially grooved for improved attachment to the feathers. The medial notches of the mandibles have sharp cutting edges for clipping the barbules for ingestion. The labrum is uniquely hinged to allow it to pull over the mandibles, closing the hypopharynx, which may be an adaptation should the louse be immersed in water. The labial palpi each have 6 terminal sensory setae. The characteristic rows of elongated setae on the posterolateral surfaces of the head, thoracic and abdominal segments would also resist dislodging during preening. The antennae have 5 segments, with the terminal segment bearing the sensory peg sensilla of the basiconica sensilla (peg organ). The 4th and 5th segments have sensilla coeloconica containing tuft organs and adjacent plate organs. These sensory sensillae enable this louse to orientate on the host as well as to locate potential mates. The stout prothorax and meso-metathorax, and well-developed fused tergopleurites which wrap around the abdominal segments, protect this louse during preening. Each of the robust legs ends in a pair of tarsal claws which interdigitate with the 3 enlarged pretarsal setae to lock onto feather barbules. An additional specialisation is the 7 rows of hooked setae on tibia II and 8 rows on tibia III which hook into the feather barbules and hold them securely against the adjacent femur surfaces. This study elucidated a number of micromorphological specialisations that enable Docophoroides to remain attached to the albatross but which may also be of taxonomic importance in future studies of these lice.

\section{Plasmodium vivax malaria in South-East Iran in 1999-2001: establishment of the responses to chloroquine in vitro and in vivo}

Y Hamedi ${ }^{\mathrm{a}}, \mathrm{M}$ Nateghpour ${ }^{\mathrm{a}}$, P Tan-ariya ${ }^{\mathrm{b}}, \mathrm{M}$ Tiensuwan $^{\mathrm{c}}$, U Silachamroon ${ }^{d}$ and S Looareesuwan ${ }^{d}$

${ }^{a}$ Department of Parasitology, School of Medicine, Hormozgan University of Medical Sciences, Bandar Abbas, Iran; and ${ }^{b}$ Department of Microbiology, Faculty of Science, ${ }^{c}$ Department of Mathematics, Faculty of Science, and ${ }^{d}$ Hospital for Tropical Diseases, Faculty of Tropical Medicine, Mahidol University, Bangkok, Thailand;yhamedi@yahoo.com

Chloroquine-resistant Plasmodium vivax is emerging in Oceania Asia and Latin America. The sensitivity of $P$. vivax to chloroquine both in vivo and in vitro in the southern part of Iran was assessed as chloroquine-resistant Plasmodium falciparum has been reported from this area. The in vitro sensitivity of $39 P$. vivax isolates was assessed. The mean $\mathrm{IC}_{50}$ and $\mathrm{IC}_{90}$ were 189 and $698 \mathrm{ng} / \mathrm{m}$ lblood, respectively; for in vivo testing, all 39 vivax malaria patients were treated with a standard regimen of chloroquine and followed-up at 28 days. The mean parasite clearance time was $67.2 \pm 22.5$ hours. The in vitro development of young parasites to mature schizonts in standard test medium was compared with that obtained in McCoy's 5A medium: no significant difference was observed. Synchronisation of the blood-stage parasites was performed according to Lambros' method but the method was unsuitable because it was detrimental to the parasites. In a number of in vitro tests performed using both our own laboratory pre-dosed microplates and WHO microplates, there was no significant difference between the results.

\section{Intestinal parasites of brown rats (Rattus norvegicus) in southern of Iran}

Y Hamedi and M Haidari

Bandar Abbas School of Medicine, Hormozgan University of Medical Sciences, PO Box 79145-4188, Bandar Abbas, Iran; yhamedi@yahoo.com

Rats are the most common animals in Bandar Abbas and its surrounding areas. According to ritual beliefs in Iran, they are 'dirty and unclean'. Therefore, we decided to determine their role as a risk factor to human and livestock health with regard to the transmission of zoonotic parasites.

The aim of the study was to determine the prevalence of infection by intestinal protozoa and helminths in the rats from Bandar Abbas. The study was undertaken during April and May 2003 at the Laboratory of Tropical Diseases, School of Medicine, Hormozgan University. The subjects were 63 rats trapped in 9 different urban areas in Bandar Abbas. The faeces were collected in the SAF fixative-preservative, using the formol-ether concentration technique. Slides were stained with modified Ziehl-Neelson (Acid-Fast) and also permanent Trichrome Stain.

Most of the animals studied were brown rats (Rattus norvegicus). Five species of enteric protozoa and ova of 3 helminth species were detected. The number and the corresponding percentage of infected animals were as follows: Entamoeba muris 12 (19\%), Cryptosporidium spp. $11(17.4 \%)$, Trichomonas minuta $10(15.8 \%)$, Spironucleus (hexamita) muris $4(6.3 \%)$, Blastocystis spp. $3(4.7 \%)$, Syphacia muris 7 $(11.1 \%)$, Hymenolepis diminuta $6(9.5 \%)$ and Trichuris muris $5(7.9 \%)$. Overall parasite occurrence was $87 \%(n=63)$, and $25 \%$ of the rats were infected with 2 or more different intestinal parasites.

The present study shows that rats are frequently infected with various intestinal parasites. This suggests that these animals represent a risk to human health by harbouring and transmitting zoonotic parasites.

\section{Cutaneous hypersensitivity induced in rabbits by extracts of the tick Amblyomma cajennense (Fabricius, 1787)}

\section{Hlatshwayo ${ }^{a}$, M J P Szabó ${ }^{b}$, G H Bechara ${ }^{b}$ and P A Mbati}

${ }^{a}$ Parasitology Research Programme, Department of Zoology, Qwa-Qwa Campus, University of the Free State, Private Bag X13, Phuthaditjhaba, 9866, South Africa; and ${ }^{b}$ Department of Animal Pathology, Faculty of Agrarian and Veterinary Sciences, State University of São Paulo, 14.870-000, Jaboticabal-SP, Brazil; hlatshwayom@qwa.uovs.ac.za

It is well known that hosts acquire resistance to tick feeding and that this resistance is immunologically mediated. In this study, the cutaneous hypersensitivity test (CHT) was used to correlate host resistance to ticks and type of reactions elicited by Amblyomma cajennense (Fabricius, 1787) tick extract in rabbits. Rabbits were divided into 3 groups of 2 animals each: naïve, pre-infested and control. CHT was induced by intradermal inoculation of $25 \mu \mathrm{g}$ extract in $0.03 \mathrm{~m} l$ of PBS in rabbit ears. Control rabbits were inoculated with PBS only. The ear thickness was measured using a Mitutoyo ${ }^{\circledR}$ device before and after $10 \mathrm{~min}$, and 1, 2, 4, 18, 24, 48, 72 and $96 \mathrm{~h}$ post-inoculation (PI). Pre-infested rabbits showed an immediate type reaction within the 1 st 10 min PI (60\% increase in ear thickness) and a delayed reaction (18 h) (85\% increase), whereas the naive rabbits showed only the immediate reaction within the 1 st $4 \mathrm{~h}$ (60\% 
increase). PBS induced only mild reactions. These results point out the crucial role of the cellular immune response of rabbits in the expression of resistance to $A$. cajennense.

\section{Trends in malaria control in South Africa: 1971-1993}

\section{L Johnson and D Moonasar}

National Department of Health, Sub Directorate Vector-Borne Diseases, Private

Bag X828, Pretoria,0001 South Africa; johnsc@health.gov.za

Malaria is a notifiable disease in South Africa and is most common in the low-altitude areas (below 1000 metres) of the Limpopo, Mpumalanga and northeastern KwaZulu-Natal provinces. Occasionally, limited focal transmission may develop in the Northern Cape and North West provinces along the Orange and Molopo Rivers. About $10 \%$ (4 million) of the total population of South Africa lives in a malaria risk area. The National Department of Health in close collaboration with the provincial malaria control programmes is responsible for monitoring malaria trends in South Africa Changes in malaria trends may result in policy change and malaria control intervention. The primary goal of the National Malaria Control Programme is to prevent mortality and to reduce morbidity. Two core indicators are used for monitoring the epidemiological trends in South Africa, namely malaria cases and deaths. In addition to the core indicators, the case fatality rate is an important indicator for monitoring case management. It may indicate possible treatment failure and late treatment-seeking behaviour. Total reported malaria cases and deaths have been recorded from 1971. The number of reported malaria cases ranged from 331 (in 1973) to 64622 (in 2000) while the number of malaria deaths ranged from 1 (in 1977) to 458 (in 2000). Following the great increase malaria cases and deaths in 2000 , the number of cases and deaths decreased steadily. Several factors such as drug and insecticide resistance, inter-country collaboration, changes in reporting system, changes in control interventions, human migration and weather patterns may explain the malaria trends and patterns over the past 22 years. These factors are discussed.

\section{Cristispira spp. associated with the digestive tracts of bivalve molluscs}

\section{$\mathrm{J} J$ Joubert and J F Eloff}

Department of Medical Virology, Faculty of Health Sciences, University of Stellenbosch, Tygerberg Campus, Private Bag X4, Gordons Bay, 7151, South Africa; johanjoub@mweb.co.za

Large spirochaetes were first described in the digestive tract, and especially the crystalline styles, of lamellibranch molluscs in 1882. They have been reported from bivalves found off the Atlantic coast of North America, the Mediterranean coast and also in freshwater lakes in Europe. No reference was found to the occurrence of these organisms in South African waters and nothing is known about their influence on the host. A number of bivalve molluscs were collected from various localities along the Southern African coastline, as well as several locations in freshwater, and examined for the presence of spirochaetes. Pinna bicolor and Streptopinna saccata collected in the offshore waters of Inhaca Island, Mozambique, were found to be heavily infected with large spirochaetes, possibly Cristispira pectinis These were mostly found in the crystalline styles. In fixed material they appeared as parallel wavy lines embedded longitudinally in the matrix of the style. In smears of dissolved styles the organisms assumed twisted shapes indicating extreme flexibility of the cells. These cells showed considerable variation in size, ranging in length between $23 \mu \mathrm{m}$ and $82 \mu \mathrm{m}$ with an average length of $44.4 \mu \mathrm{m}$ and tapering towards the end. The number of complete spiral turns per cell varied from 4 for small to 11 for exceptionally long individuals. Cells could be stained easily with $0.5 \%$ methylene blue, Hucker's ammonium oxalate crystal violet or Giemsa stain. Several nuclei could be observed along the length of the cells. Axial filaments, consisting of bundles of flagella were evident when cells from freshly dissolved styles were treated with trypsin before staining.

The frequent association of Cristispira spp. with Pinna bicolor taken from the muddy water at Inhaca Island is in accordance with previous observations by other authors who also found a higher prevalence of the organisms in oysters growing in muddy water.
The role of these organisms in the digestive tract of lamellibranchs is by no means clear. They may be either parasites or commensals. Their invariable presence in $P$. bicolor could even be indicative of a symbiotic relationship.

\section{Identification of cercariae in South Africa and Botswana}

\section{P H King}

Department of Biology, Medical University of Southern Africa, PO Box 139, MEDUNSA, 0204, South Africa; pking@medunsa.ac.za

Cercariae shed by freshwater snails have long been the cause of many important medical and veterinary diseases. The best-known examples would be fork-tailed cercariae (brevifurcate-apharyngeal distome) shed by Bulinus africanus and Biomphalaria pfeifferi and developing into Schistosoma haematobium and S. mansoni, respectively. The purpose of identifying cercariae is to enable parasitologists and veterinarians to easily confirm possible parasitic diseases in man and animals and to locate the source of infection in nature. Snails are sampled with metal scoops and placed individually in separate glass containers in a well-lit room for the spontaneous release of cercariae to take place. These cercariae are then studied alive by light microscopy using vital stains like Nile blue sulphate and neutral red to enhance their characteristics and also with scanning electron microscopy using standard techniques.

Cercariae can be classified into 3 groups based on the morphology of the tail, namely no-, single- or fork-tailed. Fork-tailed cercariae can be divided into several groups based on the following 3 features: (a) short tail rami (brevifurcate) compared to long-tailed rami (longifurcate), (b) presence of a pharynx (pharyngeal) compared to no pharynx (apharyngeal), and (c) presence of an oral sucker only (monostome) compared to the presence of both suckers (distome). A combination of these factors allows cercarial placement into clear-cut parasite groups.

Single-tailed cercariae may be divided into several groups based on one or more of the following characteristics: (a) the position and number of suckers, (b) a collar with spines around the oral sucker, (c) a spine protruding above the oral sucker, and (d) the presence of finfolds on the tail and eyespots.

When this classification of cercariae is used, parasitologists can more easily identify the source of infections caused by specific trematode parasites. Breaking the life cycle through eradication of the 1st intermediate snail hosts can then prevent long-term infections.

\section{Field assessment of non-target effects of selected cattle anti-parasiticides on dung beetles}

\section{U Kryger and C H Scholtz ${ }^{\mathrm{b}}$}

Department of Zoology and Entomology, University of Pretoria, Pretoria, 0002 South Africa; ${ }^{a} u k r y g e r @ z o o l o g y . u p . a c . z a,{ }^{b}$ chscholtz@zoology.up.ac.za

Many antiparasitic drugs used by cattle farmers may have adverse effects on beneficial dung-dwelling organisms such as dung beetles. Since the dung-burying activity of dung beetles is important for general veld conditions as well as for parasite control, it is crucial to identify antiparasitic drugs with ecotoxicological effects on dung beetles. We investigated the dung beetle compatibility of 2 different cattle antiparasitic products of the pharmaceutical company Novartis, namely Acatak ${ }^{\circledast}$ (fluazuron $2.5 \%$ pour-on) and Cevamec ${ }^{\circledR}$ (ivermectin $1 \%$ injectable). In a large-scale field study on 2 farms in the Free State province of South Africa, 2 herds of 25 cattle each were treated with a combination of both drugs and 2 additional herds remained untreated to serve as controls. The treatments were applied following the manufacturer's instructions over 1season of 9 months in 2001/02. Over a period of 11 months, dung beetles in the treatment and control camps were sampled and identified. The structure of the dung beetle communities of treated and untreated pastures were compared by calculating various measures of species richness, abundance and evenness. The distributional properties of the dung beetle communities of the different pastures were compared by means of special cumulative rank abundance plots, so-called $k$-dominance curves. In order to check for more subtle changes in species composition and/or abundance, non-metric multidimensional scaling was performed on the 4th-root trans- 
formed mean abundances of species in the control and treatment communities using the Bray-Curtis coefficient. The significance of differences between regimes (treatment - control), farms and sampling dates was established with an analysis of similarity (ANOSIM). Particular species causing similarities/dissimilarities between compared communities were identified using the 'similarity percentages' procedure (SIMPER).

All these analyses consistently indicated no difference between the dung beetle communities from treated versus untreated pastures. This suggested no adverse non-target toxicological effects of Acatak ${ }^{\circledR}$ and Cevamec ${ }^{\circledR}$ on dung beetles.

\section{The impact of resistance on malaria epidemics in KwaZulu-Natal}

\section{R Maharaj}

Medical Research Council, PO Box 70380, Overport, 4067 South Africa; raj.maharaj@mrc.ac.za

Within the ambit of malaria control, resistance to drugs and insecticides has to be examined since both types of resistance have an impact on the success of malaria control interventions. Despite a well-established malaria control programme being in place for the past 50 years, malaria epidemics still occur frequently in South Africa. The last, and most serious, epidemic since malaria control was implemented, occurred in the 1999-2000 malaria season. This study investigated the link between drug resistance and malaria morbidity in KwaZulu-Natal. Reports of drug resistance in the literature were analysed in relation to changes in malaria morbidity. The focus of this study was on the role that resistance played in the great increase in morbidity and mortality in KwaZulu-Natal in the recent epidemic. Data collected by the malaria Control Programme in KwaZulu-Natal, pre- and post-epidemic, were analysed in light of the resistance that was found in the province. During the period under review, the number of reported malaria cases increased by $380 \%$ compared to 1996 . It was determined that the epidemic was fuelled by the use of an ineffective insecticide and an inadequate drug. Resistance of more than $20 \%$ to the pyrethroid insecticide used was detected in the province as well as a $68 \%$ resistance to sulfadoxine-pyrimethamine which was used as the drug of choice for treatment. With the reintroduction of DDT and the implementation of a new drug regimen, there has been a $91 \%$ reduction in malaria morbidity in KwaZulu-Natal.

\section{The responsibility of Industry towards parasite resistance}

\section{F S Malan}

Intervet (Pty) Ltd, Private Bag X2026, Isando, 1600 South Africa;

francois.malan@intervet.com

Parasite resistance to almost all of the active groups of antiparasitic drugs has been recorded in South Africa. In the last decade only a few unrelated novel parasiticides were launched in the country. However, numerous generic products have reached, and are still going to reach the market as the patents of parent compounds expire. The high cost (US\$230 million, McKellar, 1994, Veterinary Parasitology 54: 249-258) of developing a novel compound, together with the relatively low profit margins for production animal veterinary drugs, seriously increases the risk factor regarding profitability, thus discouraging further research. Another problem is conflict between what is necessary for reducing selection for resistance (including reduced drenching) and the immediate priority for sales people to reach targets. In order that companies achieve their financial targets, pressure is placed on sales staff, which may result in the propagation of sales at the expense of good policy regarding parasite resistance management. Eventually the end user is inundated with conflicting advice on drug usage and management programmes through the media, from parasitologists, the State, Industry and its sales personnel, private veterinarians and retail outlets. It is the responsibility of each company to have its sales team adequately trained in the use of parasiticides and the potential danger of drug resistance developing due to improper parasite management programmes. It is suggested that a workshop on drug resistance be held, similar to the Worm Resistance Workshop of 1995 in South
Africa, involving all role players to discuss the seriousness of the ever-increasing resistance problem in Southern Africa and to devise a common strategy for countering parasite resistance.

\section{Cercariae shed by freshwater snails in and around Pretoria}

\section{E B E Moema and P H King}

Department of Biology, Medical University of Southern Africa, PO Box 139, MEDUNSA, 0204 South Africa; pking@medunsa.ac.za

Freshwater snails are known to serve as 1st intermediate hosts for various trematode parasites, for example Bulinus africanus serves as a host for Schistosoma haematobium and Biomphalaria pfeifferi serves as a host for S. mansoni. The main aim of this project was to collect freshwater snails in and around Pretoria and to study their cercarial sheddings.

Freshwater snails were sampled with metal scoops from various ponds, dams and rivers in the Pretoria district. In the laboratory the snails were individually placed in separate containers for the spontaneous release of cercariae. Cercariae were studied by light and scanning electron microscopy using standard techniques.

Four species of snails were sampled. These snails secreted a total of 7 different cercariae:

- Lymnaea natalensis secreted 3 types of cercariae, namely brevifurcate-apharyngeal, monostome, fork-tailed cercaria (clinostomatid cercaria), echinostome cercariae with 27 and 43 spines in the collar, respectively, and a xiphidio cercaria.

- Bulinus tropicus secreted an amphistome cercaria (Calicophoron microbothrium).

- Biomphalaria pfeifferi secreted an unknown single-tailed cercaria.

- Ferrissia fontinalis secreted a longifurcate-pharyngeal, distome cercaria (strigea cercaria).

Digenean trematodes have complex life cycles, with rare exceptions, always involving a molluscan host. It is therefore important to study snail-parasite relationships in our immediate environment.

\section{A spatial investigation of antimalarial drug resistance in Africa}

I Moodley, C Roper and B Sharp

Medical Research Council, PO Box 70380, Overport, 4067 South Africa; moodleyi@mrc.ac.za

Malaria is a complex disease that varies widely in epidemiology. One of the reasons for this variation is the parasite's susceptibility to available antimalarial drugs, of which there is a limited number. Plasmodium falciparum has developed resistance to nearly all the currently used antimalarials, and this poses a great challenge to malaria control in Africa. The geographical distribution of resistance in Africa also varies greatly. Over time, resistance becomes established in a population and thereafter reaches a level of stability in an area. A good databank of drug-resistance information is a necessary resource if these trends are to be studied adequately. Hence the primary purpose of our work is to develop an African drug resistance data repository to describe the dynamics of antimalarial drug resistance on a spatial platform using a Geographic Information System (GIS). This allows new spatial trends of drug resistance to be studied and existing ones to be confirmed or rejected. One such example is presented here. Both published and unpublished data from 1989 to the present were collected. A pro-forma input sheet was designed and used as a template to abstract information in a systematic manner. The pro-forma input sheet was designed to capture information such as survey location (geo-referenced), name and dosage of the drug studied, study methodology, treatment success or failure, therapeutic response and parasitological response. All survey results were identified by a unique identifier called a data point that allows specific information to be accessed and manipulated from the repository. To date, 307 data points have been collected from 25 countries in Africa. These data points have been incorporated within a GIS and illustrated spatially. In the present study, evidence to support the previously determined sequential emergence pattern of drug resistance in Africa is presented spatially. 


\section{A study on the morphological measurements of a Diplozoon sp. from the gills of Labeo umbratus in the Vaal River System}

\author{
L Seddon and A Avenant-Oldewage \\ Department of Zoology, Rand Afrikaans University, PO Box 524, Auckland Park, \\ Johannesburg, 2006 South Africa; ao@na.rau.ac.za
}

Few Diplozoon species have been discovered in Africa. These include Diplozoon ghanense in Alestes spp. (Ghana) and D. aegyptienses in Labeo forskalii (Egypt and Lake Albert), L. cubie (Ghana), L. victorianus (Kenya), L. cyclindricus (Tanzania) and Barilius locati (Uganda). Adult monogenean parasites range in length from less than $1 \mathrm{~mm}$ to 2 or $3 \mathrm{~cm}$. For this study, specimens were collected from the gills of Labeo umbratus in the Vaal Dam and Vaal River Barrage during a 13-month period. Following collection, the parasites were fixed in heated AFA and stored in $70 \%$ ethanol. Specimens were measured under a light microscopy after staining with boraxcarmine-iodine and clearing in lactic acid. Critical-point drying was used during preparation for scanning electron microscopy. In total, 135 parasites were collected from 50 hosts. Sixty-two parasites were measured, representing $46 \%$ of the total sample size. Data were obtained regarding the anterior length, posterior length, and total length of 57 parasites. Opisthaptor size $(n=72)$ was: length $190-1000 \mu \mathrm{m}$, width, 50-1000 $\mu \mathrm{m}$; the suckers $(n=118)$ measured: length $50-150 \mu \mathrm{m}$, width 50-150 $\mu \mathrm{m}$; and between the left and right opisthaptor clamps, 1 st clamps $(n=81)$ with length $20-110 \mu \mathrm{m}$, width $70-150 \mu \mathrm{m}$, 2nd clamps $(n=83)$ with length $30-100 \mu \mathrm{m}$, width $50-150 \mu \mathrm{m}, 3 \mathrm{rd}$ clamps $(n=82)$ with length 40-90 $\mu \mathrm{m}$, width 40-160 $\mu \mathrm{m}$, and 4 th clamps $(n=76)$ with length $10-110 \mu \mathrm{m}$, width $50-150 \mu \mathrm{m}$. Median hooks were absent. Only 7 eggs were measured and no diporpa larvae were collected. Comparisons were made between the measurements obtained during this study and measurements from other members of the genus from both Africa and Europe, and it is shown that although there is a certain measure of overlapping morphological sizes, physical structures distinguish it from other species.

\section{Pesticide resistance in arthropod pests of livestock: mechanisms, monitoring and management}

\section{G J Small}

Pest Management and Ecotoxicology Centre, Cardiff School of Biosciences, Cardiff University, Wales, UK; smallg@cardiff.ac.uk

Intensive use of organic pesticides to control arthropod ectoparasites on livestock has led to widespread selection of pesticide resistance. The main strategy for controlling populations of resistant pests has been to switch to pesticides with chemistries that are unaffected by cross-resistance. However, this has resulted in a gradual depletion in the number of pesticides available to control resistant populations as further resistance is selected. Combined with the increasing difficulty of discovering pesticides with novel chemistries and the increasing costs of bringing them to market, there is now a very major threat to the future use of pesticides in ectoparasite control. Responsibility for the development and spread of resistance has to be shared by the agrochemical companies, registration authorities and farmers. Until quite recently, agrochemica companies have placed little emphasis on resistance risk assessment for their pesticide portfolio and on managing that risk. In their defence, the registration authorities did not require them to carry out risk assessments prior to pesticide registration or to monitor and report development of resistance post-registration. Now the companies as well as registration authorities have recognised that it is in their own interests to protect the efficacy of pesticides and that they should work together to develop suitable risk-management strategies. Farmers too have contributed to the resistance problem. Some, either to reduce costs or through ignorance, have misused pesticides by not adhering to instructions for their use or by using unregistered pesticides in 'homebrews'. This has further encouraged the development of resistance. Using as examples 2 arthropod ectoparasites of South African livestock - the sheep blowfly Lucilia cuprina and the cattle tick Boophilus microplus - I will show how we have arrived at the resistance situation we face today and how strategies are now being developed to protect the pesticides still available for control.
In particular, I will introduce a new initiative to monitor and manage acaricide resistance in Boophilus species in South Africa, the South African Tick Resistance Action Committee (SATRAC).

\section{The efficacy of triclabendazole and average daily weight gain and feed conversion of feedlot cattle treated 4 and 8 weeks after artificial infections with liver fluke}

\section{Steyn ${ }^{\mathrm{a}}$, A Avenant-Oldewage $^{\mathrm{a}}$, L van Schalkwyk ${ }^{\mathrm{b}}, \mathrm{F}$ J Smit ${ }^{\mathrm{b}}$} and J S Van der Merwe

${ }^{a}$ Department of Zoology, Rand Afrikaans University, PO Box 524, Auckland Park, 2006 South Africa; and ${ }^{b}$ Virbac, Private Bag X115, Halfway House, 1685 South Africa; ao@na.rau.ac.za

Fasciolosis, or liver fluke in cattle, is a helminthosis caused by the trematode, Fasciola gigantica, which infects biliary ducts in the liver. The clinical signs of this disease in cattle are anemia, weight loss, and enteritis, resulting, in the long term, in cachexia. Anatomically, the disease is accompanied by lesions due to chronic cholangitis. In young cattle, it can have acute or sub-acute characteristics with heamorrhagic phenomena due to massive migration of the larvae. The economic impact of bovine fasciolosis is becoming even more important due to the fact that infection often develops in a pernicious way; it progresses slowly, and is not immediately recognised (HLS Veterinary Biological Products, Inc, 1999). For this reason a study was done on 30 steers, 6-8 months of age, which were artificially infected with 300 Fasciola gigantica metacercariae. The steers were placed into 3 groups of ten individuals according to their weight. The 1st group was infected with the flukes, treated at 4 weeks and again treated at 8 weeks with $12 \mathrm{mg} / \mathrm{kg}$ triclabendazole, group 3 was treated with $12 \mathrm{mg} / \mathrm{kg}$ triclabendazole at 4 weeks, and group 2 was the control group that received no treatment. Weight gain, food intake and excrement were monitored continuously. The 1 st 5 replicates in each group were selected and slaughtered 56 days after the 1 st treatment and 28 days after the 2 nd, respectively. The remaining 5 replicates in each group were slaughtered 83 days after the 1 st treatment and 55 after the 2 nd. The mean average daily weight gain was measured for all the cattle and indicated that cattle in treated groups gained more weight than cattle in the control group, and this was also the case for the calculated mean feed conversion. Liver damage was also rated on a scale of $1-5$. Cattle treated at 4 weeks still had some liver damage but no flukes. Cattle treated at 4 and 8 weeks had no flukes and the livers recovered almost completely. Therefore, triclabendazole proved to be a good treatment for fasciolosis. Repeating the treatment resulted in the cattle being in a better condition and gaining more weight.

\section{Aspects of the life-cycle of Lamproglena clariae from the Vaal River System, South Africa}

\section{A M Tsotetsi and A Avenant-Oldewage}

Department of Zoology, Rand Afrikaans University, PO Box 524, Auckland Park, Johannesburg, 2006 South Africa; ao@na.rau.ac.za

Information on developmental stages is crucial to understanding the population dynamics of any parasite. Hence, it was the aim of this study to determine the life cycle of Lamproglena clariae (Copepoda: Lernaeidae), an ectoparasite of Clarias gariepinus. The basic life stages of lernaeids as represented by Lernaea cyprinacea comprises 3 naupliar, 5 copepodite, 1 cyclopoid and adult stages. Lamproglena chinensis, however, deviates from this pattern; it has 5 naupliar and 5 copepodite stages without a cyclopoid stage. In the current study the life cycle was established through both field and laboratory observations. Adult and larval specimens of L. clariae were collected from the gills of fish in the Vaal Dam in January and April 2003. Egg sacs were removed from the adult females and incubated in the laboratory at a constant room temperature $\left(25^{\circ} \mathrm{C}\right)$ in dam water. They hatched into naupliar stages which later moulted into copepodite stages. Clarias gariepinus were infested with specimens of the 1st copepodite stages and other life stages were later collected from the hosts. These stages are described. The study showed that the naupliar and 1st copepodite stages are not the only free-living, but are also the males. Males were not attached to the host but were found moving about females attached to the host's gills. The 1st copepodite could only survive for 6 days (with no 
further development) without a host, which indicates that it is an obligatory parasite.

\section{The efficacy of triclabendazole against artificial infections of Fasciola hepatica in sheep}

\section{E van Eck, A Avenant-Oldewage ${ }^{\mathrm{a}}$, L van Schalkwyk ${ }^{\mathrm{b}}$, \\ F J Smit ${ }^{\mathrm{b}}$ and J S Van der Merwe ${ }^{\mathrm{b}}$ \\ ${ }^{a}$ Department of Zoology, Rand Afrikaans University, PO Box 524, Auckland Park 2006 South Africa; and ${ }^{b}$ Virbac, Private Bag X115, Halfway House, 1685 South Africa; ao@na.rau.ac.za}

The purpose of this study was to determine the anthelmintic effect of Flukamec ${ }^{\circledast}$ on artificial infections of Fasciola hepatica. This new treatment contains the active ingredients, triclabendazole and abamectin. Twenty-four young sheep, free of natural infections of liver fluke, were infected with viable metacercariae, collected from Lymnaea columella snails. After 4 weeks the 2 test groups were treated with a single oral dosage of, respectively, the test treatment Flukamec $^{\circledast}(10 \mathrm{mg} / \mathrm{kg}$ triclabendazole and $0.2 \mathrm{mg} / \mathrm{kg}$ abamectin $)$ and the reference treatment Fasinex (Novartis) $(10 \mathrm{mg} / \mathrm{kg}$ triclabendazole). The control group received no treatment. Inspections were carried out daily to assess the condition of the animals. Twenty-eight days after treatment, the sheep were slaughtered to recover parasites from the infected livers. The flukes were identified and counted microscopically. By comparing the average number of liver flukes recovered in the different groups, the efficacy of Flukamec ${ }^{\circledR}$ was determined. The control group had a geometric mean of 34 , while Fasinex ${ }^{\circledast}$ and Flukamec ${ }^{\circledast}$ showed a great decrease with 0.333 and 0.167 , respectively. The anthelmintic efficacy of Flukamec ${ }^{\circledR}$ was $99.5 \%$, and proved to be $0.5 \%$ more efficient than Fasinex ${ }^{\circledR}$. Under the South African system an efficacy of $>89 \%$ will qualify for a 'Control' rating as a registered product. In this study Flukamec ${ }^{\circledR}$ was very effective against 4-week-old Fasciola hepatica, and will be registered with the same efficacy rating as Fasinex (Novartis), under Act 36/1947 in South Africa.

\section{Think refugia to unlock the mental block on drug resistance}

\section{J A van Wyk \\ Department of Veterinary Tropical Diseases, Faculty of Veterinary Science, University of Pretoria, Private Bag X04, Onderstepoort, 0110 South Africa; jan.vanwyk@up.ac.za}

After the 1st documented cases in the 1960s anthelmintic resistance (AR) has escalated to the extent that in some regions in the main sheep-producing countries few worm populations and no nematicides are unaffected. However, since practically a century ago there has been ample warning that resistance was to be expected against the seemingly endless array of parasiticides developed since the 1960s. After the 1st documented case (scale, resistant to lime sulphur) in 1908, at least 12 parasite species were showing resistance by 1945 (Roush, 1990, Round Table Conference, 7th ICOPA, 197-211). In the interim, resistance has developed relentlessly, to the point that hardly any type of pest controlled by chemicals is not affected today, including bacteria, fungi, protozoa, flies, ticks, fleas, lice and even agricultural weeds.

Although there has been an avid search for alternative methods to supplement anthelmintics for more sustainable worm management, few practical systems have emerged. Totally drug-free parasite control systems are unlikely to be practical, economically acceptable, or even feasible (Van Wyk, 2002, Australian Veterinary Journal 80: 40-41). Hence existing chemicals need to be conserved (for instance by selective drenching only of animals unable to overcome the effect of reigning worm challenge) for use in situations, which demand their use. The FAMACHA ${ }^{\odot}$ system of clinical evaluation based on the colour of the conjunctivae (Van Wyk and Bath 2002, Veterinary Research 33: 509-529), is one method that has been found to be very useful for identifying for treatment, animals unable to cope with haemonchosis. In this way, all the worms in the untreated hosts are in refugia (i.e. they escape exposure to the chemicals concerned when only some of the animals are dewormed).

Notwithstanding all these warning signs from decades ago, we in veterinary helminthology failed to act on available information and persisted with control programmes that selected severely for AR, even after laboratory and field confirmation of the deleterious effect of systems such as to drench all animals immediately before a move to 'clean' pasture (containing few worms in refugia). Already in 1985 Martin's results (In: Anderson and Waller, 1985, Resistance in nematodes to anthelmintic drugs, CSIRO, Australia: 29-40) highlighted the supreme importance of refugia in selection for parasite resistance. Yet 18 years later this phenomenon is still not accorded anything close to the importance it merits, despite the severe and widespread resistance that has developed. Not only have the experts not succeeded in coming up with a strategy for alleviating the impasse, but mainly from fear of farmer intransigence, are in disagreement about the application of some of even the most basic issues. And, most incomprehensible of all, medical parasitologists are today attempting eradication or global suppression of certain human parasites, most of which are closely related to those against which much the same drugs have so dismally failed in the veterinary field.

Unless parasitologists and others recognise the central role that refugia plays in developing $\mathrm{AR}$ and cooperate to advocate and implement practical strategies which take this into account, there is no realistic hope that we will stem the rising tide of uncontrollable parasitism

\section{Worms and the resource-poor owner of small ruminant livestock in South Africa}

\section{A F Vatta ${ }^{a}$ and A L E Lindberg ${ }^{b}$}

${ }^{a}$ Onderstepoort Veterinary Institute, Private Bag X05, Onderstepoort, 0110 South Africa; and ${ }^{b}$ SWEPAR, National Veterinary Institute, SE-750 07 Uppsala, Sweden; adriano@moon.ovi.ac.za

Gastrointestinal parasitism is one of the most important disease complexes of sheep and goats impacting on the resource-poor livestock keeper. Of the responsible nematodes, Haemonchus spp., bloodsucking worms of the abomasum, pose possibly the greatest threat. Over the past several decades, the worms have been controlled through the use of anthelmintics, but the emergence of anthelmintic resistance has threatened this chemotherapeutic approach. In Africa, the overall prevalence of anthelmintic resistance has not been extensively investigated, particularly within the resource-poor farming sector. The use of stock remedies by these livestock keepers is also poorly documented in South Africa. Poor distribution networks for stock remedies and feeds within these areas, and the costs associated with purchasing these items in relation to household income currently probably limit their use. It is unlikely that methods such as intensive drenching of the whole flock will be employed by livestock owners that graze their animals communally and therefore that anthelmintic resistance would be as strongly selected for as in the commercial sector of South Africa and elsewhere. However, increases in productivity and reproduction of livestock and accompanying development of markets for sale of animals are seen by international funding bodies as a way out of poverty for communities that keep livestock. This may lead to intensification of farming and the greater need for parasite control. At such times, the risk of resistance developing to anthelmintics is much greater and there is then a need to look at alternatives to their use. Proposed strategies include the appropriate, but sparing, use of anthelmintics and the use of alternatives such as strategic protein supplementation. What is also very clear and has been stated in a number of studies is that there is a strong demand for knowledge about animal diseases, including helminthosis, and their effective management. This is an important challenge to meet and an important factor to consider in promoting appropriate helminth control strategies.

\section{The impact of parasiticide mismanagement on wildlife in South Africa}

\section{G H Verdoorn}

Poison Working Group, PO Box 72334, Parkview, 2122 South Africa; nesher@tiscali.co.za

South Africa has a respectable range of endoparasiticides and ectoparasiticides available to livestock farmers. Both classes of parasiticides have been implicated in wildlife poisoning, e.g. the loss of oxpeckers of the Boophagus genus through incompatible acari- 
cides that contain organophosphorus active ingredients. Modern products are much less harmful to wildlife in general but mismanagement of products poses a real threat to many natural biotas. The common practice of preparing home-made acaricides increases the risk of parasite resistance to active ingredients while posing a poisoning threat to beneficial taxa such as oxpeckers, starlings, cattle egrets, dung beetles and soil nematodes. Ineffective applicators result in gross under-dosing or overdosing of animals and leads to parasite resistance and wildlife impacts. Of particular concern is the use of endoparasiticides and ectoparasiticides on game animals. This compromises the natural resistance of wildlife to endo- and ectoparasites, and also results in a potential food shortage for oxpeckers and may be responsible for dung beetle mortality. While only two ectoparasiticides are legally registered on game animals in South Africa, game farmers use a variety of products, including home-made concoctions. Automatic applicators are used extensively for applying ectoparasiticides to all animals irrespective of species, size or ectoparasite loads. Endoparasiticides that are registered as oral applications or bolus applications for bovids and ovids are often mixed in with supplemental licks for ungulates as a means of deworming game animals. This practice offers no guarantee of correct dosages and can only lead to endoparasite resistance and a weakened gene pool in ungulates, and impacts negatively on beneficial organisms.

\section{Africa, a possible origin of the amphibian chytrid fungus}

\section{Weldon and L $\mathrm{H}$ du Preez}

School of Environmental Sciences and Development, Potchefstroom University,

Private Bag X6001, Potchefstroom, 2520 South Africa; drkcw@puknet.puk.ac.za

The sudden appearance of the emerging infectious disease, chytridiomycosis, and its aetiological agent, Batrachochytrium dendrobatidis, as the cause of amphibian deaths and population declines in several continents suggest that $B$. dendrobatidis was introduced into the affected regions, with the earliest record being 1974 in the USA. Although the origin of $B$. dendrobatidis is unknown, international movement of infected amphibians provides a route of transmission between nations. Owing to widespread records in Africa with no apparent major amphibian declines and a lucrative international trade in Xenopus laevis, we hypothesised that $B$. dendrobatidis had originated in Africa. A survey of 697 archived specimens of 3 species of Xenopus collected from 1890 to 1999 in southern Africa was conducted. Webbing from the hind foot was removed and processed for histology and standard $\mathrm{H} \& \mathrm{E}$ staining. We found the earliest case of chytridiomycosis from 1938. All species were infected with an average prevalence of $2.7 \%$ and a relatively stable prevalence in the decades after 1929. The number of specimens available from before 1938 was inadequate to detect infected populations with prevalences below $10.5 \%$. This result supports the hypothesis that $B$. dendrobatidis originated in Africa and was disseminated from southern Africa via trade in X. laevis.

\section{Posters}

\section{Amoebiasis prevalence at King Edward VIII Hospital in Durban - a 10-year retrospective study}

\section{B Anderson, T F H G Jackson and F Nkwanyana}

S.A. Medical Research Council, PO Box 70380, Durban, 4001 South Africa; canderson@mrc.ac.za

Amoebiasis is endemic in Durban. Between 80 and 120 deaths occur annually from this disease at King Edward VIII Hospital $(\mathrm{KEH})$. The amoebic gel diffusion test (AGDT) has been the serological tool used by the Amoebiasis Research Unit (ARU) for the diagnosis of invasive amoebiasis at KEH for the past 40 years. During the study period, all suspected cases of invasive amoebiasis investigated at KEH were tested serologically by the ARU. The AGDT has a specificity of $100 \%$ and a sensitivity of $98 \%$ in amoebic liver abscess (ALA) and $89 \%$ in amoebic dysentery (AD). This presentation was a retrospective study of the 20-hour positive results (which indicate current invasion by the pathogen) over a 10-year span, 1989 to 1999. During this period, there was a consistent decline in serological activity across the year from January to December. Annually, patients were discharged from hospital in December for the holi- days, and this could be a contributing factor in the reduced numbers presented for that month. There were twice as many amoebiasis patients diagnosed in January as in December. Over the 10-year period, the total number of patients diagnosed by the AGDT increased from 1989 to peak in 1993. They dropped greatly in 1995, and continued to gradually decline until 1998. In 1999 the numbers were similar to those found in 1989. Dividing the year into quarters, all results indicate a marked peak in numbers of positive cases in 1992 and 1993.

In conclusion, at $\mathrm{KEH}$ :

- In 1993, there was a substantial increase in the number of amoebiasis cases diagnosed.

- The highest number of amoebiasis cases appears to be diagnosed between January and April each year.

- The lowest numbers of cases are diagnosed from September to December.

- The prevalence of amoebiasis (as indicated by the AGDT) remains similar at the beginning of the study (1989) to the figures found at the end of the 10-year period (1999).

These results indicate that the presence of amoebiasis in this region has not declined significantly over the 10-year period, and suggests that the reintroduction of a cysticidal drug to be used in combination with metronidazole might be efficacious in eradicating E. histolytica from the poorer rural communities in Durban and its surrounds.

\section{Some effects of garlic on the salivary gland structure and function of feeding adult Hyalomma marginatum rufipes females}

\section{A Els, A Motlhatlhedi and S R Magano}

Department of Biology, Medical University of Southern Africa, PO Box 139, MEDUNSA, 0204 South Africa; dels@medunsa.ac.za

In view of the overall aim of this study, which was to determine whether garlic visibly influences the salivary gland structure and function of feeding female $H$. m. rufipes, the specific objectives set for this study were to describe the salivary gland structure and to investigate the possibility of a changed diaphorase activity in host-fed, and in artificially fed ticks.

Sixty-six flat adult $H$. m.rufipes ( 24 males, 42 females) were used. Four albino rabbits served as hosts (2 received drinking water supplemented with garlic and 2 pure water). Each host was infested with 6 flat females, and 6 flat males of $H$. m. rufipes. Of the flat females used during tube-feeding, 9 received garlic supplemented in water, and 9 only pure water.

Indirect supplementation or non-supplementation of garlic (through the host-feeding programme) or direct supplementation or non-supplementation (during the tube-feeding programme) provided 4 different treatment groups. Salivary gland samples were taken on 3 occasions for routine histology, ultra-thin sections, and histochemistry from each treatment group: before feeding started, after feeding the ticks for 3 days and after 5 days. Routine histology and ultra-thin sections of the salivary glands showed the development in both size and complexity of the salivary gland acini. Before the commencement of feeding the salivary gland mainly comprises type I acini, while type III acini were only observed in the salivary glands 3-5 days after feeding started. Differences in the structure and abundance of well-developed acini were evident between garlic and non-garlic treatment groups. In tube-fed ticks very few acini were observed on the 5th day of feeding. Though diaphorase activity was positively demonstrated, no marked differences were recorded between treatment groups. Differences in the acini development between garlic treatment and non-garlic treatment groups, and a slight difference in diaphorase activity between these treatment groups, indicated that garlic affected the feeding activities of adult females of $H$. m. rufipes.

\section{Sero-diagnosis of Trichomonas vaginalis}

\section{Fernandes, M P Mabille and A A Hoosen}

Department of Medical Microbiology, Medical University of Southern Africa, PO Box 204, MEDUNSA, 0204 South Africa; fernan@medunsa.ac.za

Trichomonosis, a sexually transmitted infection caused by the parasite Trichomonas vaginalis, affects both males and females. The 
males are mainly asymptomatic while females may present with a muco-purulent, frothy vaginal discharge. Because of asymptomatic carriage, infected sexual partners may continue to infect their partners, further spreading the disease. The disease may not be conclusively diagnosed based on clinical presentation alone. Because of the ever-increasing problems associated with sexually transmitted infections, and the widely held view that they also contribute to the transmission of HIV, the aim of this study was to develop an indirect immunofluorescent test for testing patient sera for antibodies to T. vaginalis antigen to identify asymptomatic cases. An indirect immunofluorescence test was developed using whole T. vaginalis parasites, fixed onto microwell slides as antigen. Serum dilutions were then tested for the presence of IgG or IgM antibodies. Out of a total of 120 patients tested, ten $(8.3 \%)$ were found to have antibodies to T. vaginalis antigen, of them 6 were found to be IgM positive while 5 were found to be IgG positive, with 1 patient positive for both IgG and IgM, the rest, $110(91.7 \%)$ were found to be negative. The presence of specific T. vaginalis antibodies in serum was demonstrated relatively easily. The antibody titres were low and antibodies of both the IgG and IgM class were demonstrated. The indirect immunofluorescence test could be used to test asymptomatic cases for the presence of $T$. vaginalis antibodies.

\section{In-vitro susceptibility testing of Trichomonas vaginalis}

\section{Fernandes and A A Hoosen}

Department of Medical Microbiology, Medical University of Southern Africa,

PO Box 204, MEDUNSA, 0204 South Africa; fernan@medunsa.ac.za

This study was undertaken to determine the emergence of resistance in T. vaginalis isolates obtained from a tertiary diagnostic hospital laboratory service using a variety of methods. In the absence of internationally acceptable susceptibility testing guidelines, questions have been raised about atmospheric requirements, time of incubation and visual detection of endpoints. In this study, minimum inhibitory concentrations (MIC) were determined for 36 isolates where: (i) aerobic incubation was compared with anaerobic incubation; (ii) incubation time of 24 hours was compared with 48 hours and (iii) endpoints were determined by use of a $\mathrm{pH}$ colour indicator for visual readings to be compared with microscopic results. Minimum lethal concentrations (MLC) results were also obtained by passaging isolates into fresh culture medium and incubating them for 7 days. All strains tested had MIC values $8 \mu \mathrm{g} / \mathrm{ml}$. For the $\mathrm{MIC}_{50}$ and $\mathrm{MIC}_{90}$ the values were lower after 24 hours incubation compared with 48 hours incubation. Visual MIC readings were slightly lower than microscope-read results for both aerobic and anaerobic incubation. Value for the MLC concentrations ranged from $0.25 \mu \mathrm{g} / \mathrm{m} l$ to $12.5 \mu \mathrm{g} / \mathrm{m} l$. The $\mathrm{MLC}_{50}$ for aerobic incubation was higher than $\mathrm{MLC}_{50}$ under anaerobic incubation, with the $\mathrm{MLC}_{90}$ for anaerobic incubation higher than $\mathrm{MLC}_{90}$ under aerobic incubation. All strains tested were regarded as susceptible to metronidazole (commonly agreed resistant values being $100 \mu \mathrm{g} / \mathrm{m} \ell$ ). Recommended conditions for susceptibility testing will be a 24-hour incubation period under aerobic conditions with the results being read using a microscope.

\section{Karyotyping of Trichomonas vaginalis}

\section{Fernandes and A A Hoosen}

Department of Medical Microbiology, Medical University of Southern Africa,

PO Box 204, MEDUNSA, 0204 South Africa; fernan@medunsa.ac.za

Although Trichomonas vaginalis is responsible for the world's most common nonviral sexually transmitted disease, little is known about the differences between strains. There is reason to believe that the human genitourinary trichomonad consists of different types, possibly even different subspecies, on which the pathology and clinical course depend. The separation of intact chromosomal DNA was performed by Pulse Field Gel Electrophoresis (PFGE) to determine the molecular karyotype of 7 strains of $T$. vaginalis using the CHEF-DR PFGE system. A total of 6 chromosomes, with no difference in banding pattern among the 7 isolates, were separated, which fell into 3 distinct size classes. The 1 st class contained 1 ' mini' chromosome of approximately $75 \mathrm{kbp}$ in size. The 2nd class contained 2 'intermediate' chromosomes of about $1100 \mathrm{kbp}$ and $1200 \mathrm{kbp}$ in size, and the last class contained 3 'maxi' chromosomes of approximately $3500 \mathrm{kbp}, 4700 \mathrm{kbp}$ and $5700 \mathrm{kbp}$ in size. The method presented in this study can be regarded as an improvement on the only other published method, since it is more cost-effective, using fewer reagents, and has a much shorter run time.

\section{Sexual dimorphism in the albatross louse Harrisoniella hopkinsi (Eichler, 1952)}

\section{E D Green ${ }^{\mathrm{a}}$ and M L Turner}

${ }^{a}$ Department of Anatomy and ${ }^{b}$ Electron Microscope Unit, Medical University of Southern Africa, PO Box 232, MEDUNSA, 0204 South Africa;

edward@medunsa.ac.za

Harrisoniella hopkinsi is very large louse that infests the wing feathers of the large albatross species. Although both sexes measure approximately $9 \mathrm{~mm}$ in total length, the males are characterised by greatly enlarged antennae compared to the filiform antennae of the females. These differences were so great that the phthirapterist Kellog described the nymphs and adult males as separate species in 1914. Additional sexually dimorphic characteristics were observed using both light and scanning electron microscopy (SEM). The lice were collected from nesting wandering albatrosses at Dunedin, New Zealand, and fixed in $70 \%$ ethanol. After ultrasonication, the lice were routinely prepared for SEM and viewed in a Leica Stereoscan 420 SEM at 5 to $10 \mathrm{kV}$. Compared to the tubular filiform antennae of the female, the large antennae of the male are further specialised in the following ways. The 1st 3 proximal segments are greatly enlarged and elongated in an anterior direction. The 1 st segment has a lateral toothlike process while the 3rd segment terminated as a hook-like process. The distal 2 antennal segments of both sexes are similar and contain the typical sensoria. The head of the male has a clypeal signature that forms a dorsal prominence, which is poorly developed in the female. The posterior parietal margins of the head of the female are distinctly more bulbous than those of the head of the male. The eye lenses of the female are also larger than those of the male. Tergites 1 to VII in the male are fully sclerotised while tergites I to VI in the female are sclerotised laterally but separated by a medial non-sclerotised band. The terminal sternites form the subgenital plate in the female while in the male the terminal abdominal segment forms the funnel-shaped terminalia which has an arrangement of setae that is speciesspecific. While some sexual dimorphism of the antennae and terminalia is common in this family Philopteridae (feather lice), the magnitude of the morphological differences between $H$. hopkinsi males and females is unique.

\section{In vitro monitoring of Plasmodium vivax sensitivity to chloroquine in Thailand}

\section{Y Hamedi ${ }^{a}$, M Nateghpour ${ }^{a}$, B Soonthornsata ${ }^{b}$, P Tan-Ariya ${ }^{c}$,}

S Kojima ${ }^{d}$ and S Looareesuwan ${ }^{b}$

${ }^{a}$ Department of Parasitology, Bandar Abbas School of Medicine, Hormozgan University of Medical Sciences, Bandar Abbas, Iran: ${ }^{b}$ Department of Clinical Tropical Medicine, Faculty of Tropical Medicine, Mahidol University, Bangkok, Thailand; and ${ }^{c}$ Department of Microbiology, Faculty of Sciences, and ${ }^{d}$ The Asian Centre of International Parasite Control, Mahidol Faculty of Tropical Medicine,

Bangkok, Thaiand; yhamedi@yahoo.com

Plasmodium vivax is the second most common cause of malaria. Chloroquine (CQ), a 4-aminoquinoline antimalarial has been the drug of choice for the treatment of vivax malaria for more than 40 years in many parts of the world. CQ is well absorbed, well tolerated and inexpensive; $\mathrm{CQ}$ rapidly and effectively eradicates blood-stage parasites, usually within 36-72 hours. However, in the past decade CQ-resistant $P$. vivax has been found in several countries; the 1 st indication that $P$. vivax might be developing resistance to $C Q$ was the failure of an 8-month-old infant to respond adequately to treatment with chloroquine in Papua New Guinea; subsequent reports have provided further confirmation of the presence of $C Q$-resistant vivax malaria in the Southwest Pacific, Indonesia, Brazil, Myanmar and, recently, Colombia.

Malaria is an important infectious disease in Iran; its transmission is prevalent in 3 of the country's southeastern provinces. Whereas the in vitro and in vivo resistances of $P$. falciparum to CQ have been reported from Iran, the in vitro sensitivity of $P$. vivax to CQ had not been established there. The in vitro sensitivity of Plasmodium vivax to chloroquine was investigated in patients admitted to the Bangkok Hospital for Tropical Diseases. Of 42 isolates, 34 cases were successfully tested for in vitro parasite sensitivity to chloroquine. The results showed a significant decrease in sensitivity compared with data 
published in 1989 and 1995: the $\mathrm{IC}_{50}$ and $\mathrm{IC}_{90}$ were 187.2 and $1217.9 \mathrm{ng} / \mathrm{m}$ l blood, respectively.

There is approximately a 4 -fold decrease in sensitivity compared with other data gathered during the past 2 decades. A number of in vitro tests were performed using both WHO microplates and our own laboratory-predosed microplates, simultaneously, under the same conditions. There was no significant difference between the results.

\section{Prevalence of Trypanosoma lewisi in rats in the southern parts of Iran}

\section{Y Hamedi ${ }^{\mathrm{a}}$ and $\mathrm{M}$ Haidari $^{\mathrm{b}}$}

${ }^{a}$ Bandar Abbas School of Medicine, ${ }^{b}$ Bandar Abbas School of Public Health,

Hormozgan University, Bandar-Abbas,Iran; yhamedi@yahoo.com

The aim of this study was to determine the prevalence of Trypanosoma lewisi among rats in Bandar-Abbas city. Trypanosoma (Herpetosoma) lewisi is a trypanosome of the subgenus Herpetosoma (Stercoraria subdivision), a blood flagellate parasite of rats (Rattus rattus and Rattus norvegicus) that is transmitted by fleas. Rats are infected primarily via the oral route following the ingestion of infected fleas or rat food contaminated by flea faeces containing metacyclic trypanosomes. All trypanosomes of the subgenus Herpetosoma are non-pathogenic to their vertebrate hosts.

This study was undertaken during April and May 2003 at the Faculty of Medicine, Hormozgan University. The subjects were 63 rats, which were trapped in different areas in the city and transferred to the laboratory. They were anaesthetised with ether, the species and sex were recorded and the ectoparasites collected. Finally, 2--3 me blood was taken directly from the rats' hearts, from which thick and thin blood films were prepared. Diagnosis of T. lewisi in the rats was made by microscopic observation of Giemsastained blood smears.

The most prevalent wild rats were Rattus norvegicus and the second most common ones were Rattus rattus. Most of the fleas examined were Xenopsylla cheopis, and 9 of 63 rats were positive for Trypanosoma lewisi (infection rate: $12.7 \%$ ). The other vector-borne organisms, which were observed in Giemsa-stained blood films, were Babesia muris, Borrelia spp. and Anaplasma spp. (order Rickettsiales), the latter being transmitted by ixodid ticks.

Regarding the high prevalence of Trypanosoma lewisi among the wild rats, this stercorarian species can be used as a model for different aspects of trypanosomosis , though there are no cases of sleeping sickness in Iran.

\section{The effect of Amblyomma cajennense and Rhipicephalus sanguineus saliva on the in vitro proliferative responses of T lymphocytes from BALB/c mice}

\section{Hlatshwayo ${ }^{a}$, B R Ferreira ${ }^{\mathrm{b}}$ and P A Mbati ${ }^{\mathrm{a}}$}

${ }^{a}$ Parasitology Research Programme, Qwa-Qwa Campus, University of the Free State, Private Bag X13, Phuthaditjhaba, 9866 South Africa; and ${ }^{b}$ Department of Immunology and Biochemistry, School of Medicine of Ribeirao Preto, University of São Paulo, 14049-900, Ribeirao Preto-SP, Brazil; hlatshwayom@qwa.uovs.ac.za

Tick saliva has been shown to contain a variety of pharmacologically active molecules, including those with immunosuppressive characteristics. There is increasing evidence that the non-specific suppression of host immunity by tick saliva is exploited by tickborne pathogens, e.g. the saliva-activated transmission of some tick-borne viruses. Several studies have revealed that T-lymphocytes and cytokines play a crucial role in determining the outcome of parasitic infections in terms of protective immunity. In this study, we investigated tick saliva effects on T-cell proliferation, an event associated with host immune defence mechanisms. The proliferative response of lymphocytes from BALB/c mice exposed to the saliva of Amblyomma cajennense (Fabricius 1787) and Rhipicephalus sanguineus (Latreille, 1806), were therefore compared in vitro. We determined that $A$. cajennense tick saliva inhibited Con-A induced splenic T-cell proliferation. Tick saliva diluted twenty times (75 $\mu \mathrm{g} / \mathrm{m} \ell$ ) inhibited Con-A induced T-cell proliferation by $91 \%$. Rhipicephalus sanguineus tick saliva diluted twenty times $(64 \mu \mathrm{g} / \mathrm{m} l)$ also inhibited Con-A induced splenic T-cell proliferation by $56 \%$. These data suggest that the facilitating effect of saliva on the establishment and transmission of some tick-borne pathogens might be associated with the suppression of the host innate resistance mechanisms.

\section{The effect of plant extracts on host resistance to tick infestation}

M Moshe, S R Magano and D A Els

Department of Biology, Medical University of Southern Africa, PO Box 139, MEDUNSA, 0204 South Africa; smagano@medunsa.ac.za

Much attention in recent times has been devoted to the exploitation of acquired resistance to tick infestation by hosts as an alternative non-acaricidal tick control measure. Apart from our current work which investigates the effects of garlic (Allium sativum) extracts on tick-host interactions, no other work is known to us in which extracts of plants that have the capacity to modulate immunity have been tested using tick-host models. In this study, rabbits that were used as tick hosts were divided into treatment $(n=5)$ and control $(n=5)$ groups. The treatment group $(n=5)$ was fed water mixed with garlic extract and rabbit pellets while the control group $(n=5)$ was fed water and rabbit pellets only. Each rabbit in both the treatment and control groups was subjected to 2 successive infestations with adults of Hyalomma marginatum rufipes ticks. Engorgement weight of ticks in the 2 groups and levels of blood cell types and blood proteins were monitored to determine the effects of garlic on ticks and on the host. Our results so far suggest that garlic extracts increase white blood cell count in rabbits. However, the feeding performance of ticks (assessed as tick engorgement weight) appeared not to be affected by garlic. No significant differences between the 2 groups were found in blood protein results.

\section{Developing methods for the screening of ethnoveterinary plants for tick control}

\section{F Nchu ${ }^{a}$, S R Magano and J N Eloff ${ }^{\mathrm{b}}$}

${ }^{a}$ Department of Biology, Medical University of Southern Africa, PO Box 139

MEDUNSA, 0204 South Africa; and ${ }^{b}$ Department of Pharmacology and Toxicology, University of Pretoria, Pretoria, 0001 South Africa; smagano@medunsa.ac.za

Ticks and the diseases they transmit to man and other animals hamper successful livestock production and interfere with the economic growth of a country, particularly in Africa. The current tick control methods rely on the use of acaricides for dipping or spraying livestock and are as a result fraught with many problems, including the emergence of acaricide-resistant strains in tick populations. These problems raise the need to develop alternative approaches to tick control that exclude or reduce the requirements for acaricide use. In our study, bioassays for the repellency and toxicity of crude extracts of Allium plant species to adults of Hyalomma marginatum rufipes are described. Dichloromethane, acetone, ethanol and distilled water were used as solvents to make plant extracts. The repellency index for each extract was calculated using the formula $R=\left[\left(N_{\mathrm{c}}-N_{\mathrm{t}}\right) /\left(N_{\mathrm{c}}+N_{\mathrm{t}}\right)\right] \times 100$ where $N_{\mathrm{c}}$ is the number of ticks on the control rod and $N_{\mathrm{t}}$ is the number of ticks on the treatment rod. Among the 3 Allium species (A. sativum, A. porrum and A. cepa) investigated in this study, acetone extracts of $A$. porrum showed a high repellency index (mean range: 65-79.48\%). The toxicity bioassay showed $100 \%$ mortality in ticks that were in contact with dichloromethane extract of $A$. sativum within an hour. This toxicity persisted to the 2 nd day. The results obtained in this study presents Allium plants as possible sources of agents that can be used to control ticks.

\section{Impact of environmental conditions on the prevalence of intestinal parasites in the Durban metropolitan area}

\section{A F Sumad, C B Anderson and T F H G Jackson}

South African Medical Research Council, PO Box 70380, Overport, 4067 South Africa;sumadf@mrc.ac.za

In many parts of the world, public health infrastructure does not exist, and water- and food-borne diseases remain major causes of morbidity and mortality. Viral, bacterial and parasitic diseases cause illness by a variety of means that overwhelm the immune system. Health professionals are realising with increasing frequency that the detrimental impact and far-reaching implications that intestinal parasites have upon the lives of people in impoverished communities. It is now known that chronic worm infestations can down-regulate the immune response necessary to prevent infections such as 
HIV, TB and probably other diseases; and that vaccine efficacy can be compromised by endemic worm infestation. Our study was performed on adult members of 100 families living in informal settlements in either rural or urban areas of the Durban Metropolitan region. A Geographical Information Systems (GIS) method was developed to examine the relationship between water supply, sanitation and intestinal parasites (note that 'parasites' includes malaria, Theileria, Leishmania, etc.). Faecal analysis, including formol-ether concentration of stools and Robinson's culture for the isolation of amoebae, indicated the presence of pathogens that included Entamoeba histolytica, Giardia lamblia, Trichuris trichiura, Ascaris lumbricoides, hookworm species, Schistosoma mansoni, and Taenia species in this population during a 1-year period. Prevalence of intestinal parasites was higher in the rural areas and informal settlements than in more affluent suburban areas. The level of public health infrastructure and delivery influenced the prevalence of intestinal parasites. To reduce transmission of these organisms, alleviation of poverty and health-promoting activities are advocated to help change cultural and behavioural practices.

\section{An analysis of malaria cases occurring at the Ga-Rankuwa Hospital over a 5-month period in 2003}

\section{K SweSwe, D Ngwira, P P Sein and A A Hoosen}

Department of Medical Microbiology, Medical University of Southern Africa,

PO Box 211, MEDUNSA, 0204 South Africa; psein@medunsa.ac.za

This study was undertaken to analyse malaria cases seen at Ga-Rankuwa Hospital over a 5-month period, January to May 2003, and to compare the results with the previous study done from November 1997 to May 1998. Data that include sex, age, clinical presentation, travel history, chemoprophylaxis, treatment and outcome were collected for all laboratory-confirmed malaria cases. The total number of laboratory-confirmed malaria cases was 40 . There were 19 males, 13 females and 4 children with age ranges of 20-56 years, $28-41$ years and 6 months -7 years, respectively. Thirty-five patients presented headaches, fever, chills and myalgia; 1 patient presented severe anaemia, diarrhoea and genital ulcer. For the 4 patients that were seen at the outpatient's department, clinical data were not available. The majority of patients had visited Mozambique and none of them had taken chemoprophylaxis. From the blood specimens received on admission (156 in total), Plasmodium falciparum antigen was detected in 55, but $P$. falciparum trophozoites were seen in 40 patients. Parasitaemia ranged from $0.001-23.5 \%$. Thirty-four patients were treated with quinine only, 5 patients with quinine and doxycycline, no patients were on chloroquine and 1 patient died before initiation of antimalarial therapy. Over the 5-month study period, 40 malaria cases were confirmed. In the previous study done over a 7-month period there were 37 confirmed cases. The majority of cases were also imported from the neighboring southern African countries, mainly Mozambique. In this study no patient presented with cerebral malaria while in the previous study 3 patients were treated for cerebral malaria. In both studies none of the patients took chemoprophylaxis. In the previous study, chloroquine was administered to 6 patients while in the current study none received chloroquine. This is in keeping with the current recommendations. The patient who died was a 27 -year-old male who had an unusual presentation at admission, i.e. fever, severe anaemia, chronic diarrhoea for 2 months and genital ulcer, and this might have led to the late diagnosis of malaria. This patient's peripheral blood film had all the poor prognostic features such as the presence of all stages of $P$. falciparum, including immature and mature schizonts, malaria pigments in white blood cells and very high parasitaemia $(23.5 \%)$.

\section{Field efficacy study of 2 proprietary anthelmintics against natural infections of nematode parasites in impala (Aepyceros melampus)}

\section{J S Van der Merwe ${ }^{a}$, F J Smit ${ }^{\mathrm{b}}$, T Ahlers ${ }^{\mathrm{c}}$, J P Louw ${ }^{\mathrm{d}}$ and}

\section{A M Durand ${ }^{\mathrm{e}}$}

${ }^{a}$ Virbac RSA, Private Bag X115, Halfway House, 1685 South Africa; ${ }^{b}$ PO Box 4511, Mokopane, 0600 South Africa; 'Mabalingwe Game Ranch, Bela-Bela, 0480 South Africa; ${ }^{d} 184$ Marija Street, Wonderboom, Pretoria, 0182 South Africa; and ${ }^{e}$ PO Box 18348, Pretoria North, 0116 South Africa; kvandermerve@virbac.co.za

This study was designed to evaluate the efficacy of an abamectin premix and a very long-acting ivermectin against the natural infections of nematode parasites of impala. The game industry is in need of registered anthelmintics. Currently, large volumes of anthelmintics for domestic ruminants are being used extra-label for game. Registration authorities require validation of a product in the target animal. Healthy impala in 1 family group were captured by experienced game rangers in an ethical way, minimising heat and other stress. The 2 treatment and 1 control groups consisted of 20 animals. The Treated ${ }_{A}$ group ( 6 animals) received the abamectin premix at a dose rate of $1 \mathrm{~g} / 90 \mathrm{~kg} / \mathrm{day}$ in the feed for 7 days. The Treated $(6$ animals) group was injected subcutaneously with the long-acting ivermectin at a dose rate of $1 \mathrm{~m} / 50 \mathrm{~kg}$ body mass. The control group (8 animals) was left untreated. The numbers of each nematode parasite species recovered were counted and the geometric means of treated and untreated groups used to determine anthelmintic efficacy. The abamectin premix showed $100 \%$ efficacy against Haemonchus contortus, Impalaia spp., immature Cooperia, Oesophagostomum spp. and adult Bigalkenema spp. It was less effective against adult Cooperia spp. and adult Trichostrongylus spp.

The very long-acting ivermectin was $>90 \%$ effective against all nematode parasites found in the impala. From the results obtained, registration of this formulation as an anthelmintic for use in impala seems to be the option to pursue.

\section{Field tick efficacy study in buffalo with an amitraz/cypermethrin pour-on}

\section{J S Van der Merwe ${ }^{\mathrm{a}}$, F J Smit ${ }^{\mathrm{b}}, \mathrm{H} \mathrm{Heyne}^{\mathrm{c}}$ and A M Durand ${ }^{\mathrm{d}}$} ${ }^{a}$ Virbac RSA, Private Bag X115, Halfway House, 1685 South Africa; ${ }^{b}$ PO Box 4511, Mokopane, 0600 South Africa; ' $P O$ Box 12532, Onderstepoort, 0110 South Africa; and ${ }^{d}$ PO Box 18348, Pretoria North, 0116 South Africa;

kvandermerve@virbac.co.za

The game-farming industry has grown in recent years in South Africa with a concomitant increase in relocation of animals and breeding programmes, especially for disease-free buffalo (Syncerus caffer). Parasite control may be essential to reduce stress levels, physical damage inflicted by parasites and the transmission of tick-borne diseases. A $1 \%$ amitraz/cypermethrin pour-on formulation, registered for use in cattle in South Africa, was evaluated as a potential acaricide for control of ticks in buffalo. The efficacy of the formulation against a natural tick infestation was evaluated. Sixteen buffalo, not subjected to ectoparasite treatment for 3 weeks before commencement of the trial, were involved in the investigation. The buffalo were not ranked and allocated to the 2 groups according to any criteria, but were treated at random to reduce handling stress. Eight animals were treated with the pour-on formulation to a dose rate of $0.1 \mathrm{~m} / \mathrm{kg}$ body mass. Eight animals formed the untreated control group. The required dose for each animal was applied along the dorsal midline area from the withers to the tubae coxae. At the end of the study the buffalo were returned to the game reserve. No chemically induced adverse reactions were observed in any of the animals throughout the trial. The efficacy of the pour-on was $94.6 \%$ against Amblyomma hebraeum, $100 \%$ against Rhipicephalus evertsi evertsi, $99.9 \%$ against Rhipicephalus appendiculatus and $100 \%$ against Hyalomma marginatum rufipes.

\section{The efficacy of an amitraz/cypermethrin pour-on applied topically against the lice of ostriches (Struthio camelus)}

\section{J S Van der Merwe ${ }^{a}$, F J Smit ${ }^{b}$, L Van Schalkwyk ${ }^{c}$ and A M Durand ${ }^{d}$ \\ ${ }^{a}$ Virbac RSA, Private Bag X115, Halfway House, 1685 South Africa; ${ }^{b}$ PO Box 4511, Mokopane, 0600 South Africa; ${ }^{\circ}$ Bosvet Clinical Development (Pty) Ltd, Suite 8396, Private Bag X32, Kempton Park, 1620 South Africa; and ${ }^{d}$ PO Box 18348, Pretoria North, 0116, South Africa; kvandermerve@virbac.co.za}

This study was designed to evaluate the efficacy of a $1 \%$ amitraz/cypermethrin formulation applied topically at $1 \mathrm{~m} / 10 \mathrm{~kg}$ to ostriches against Struthiolipeurus struthionis. Ostriches harbouring infestations of lice were selected and left for 25 days to allow the louse population to increase. Nine ostriches were involved in this trial; the treatment group consisted of 6 ostriches and the control group of 3 . The ostriches were approximately 6 months old. Accepted statistical procedures were used to select the treatment and untreated control groups. The treatment group was treated on Day 0 with the pour-on as follows: $1 \mathrm{~m} \ell$ on each wing, $2 \mathrm{~m} \ell$ on the 
back, $1 \mathrm{~m}$ lon the tail and $0.5 \mathrm{~m}$ lon the neck. Half the remaining dose was applied to each thigh. Application was done in this manner as topical remedies are known not to disperse well on ostriches. The ostriches in the control group remained untreated. When the birds were slaughtered, feathers from different parts of the body were collected separately, placed in plastic bags and weighed to determine the total weight of feathers per body part. The skin was examined macroscopically for the presence of lice. Lice found were collected and identified. 1/10 aliquots were collected from the feather samples and lice present counted under a stereo microscope. Acaricidal efficacy was determined. No drug-related adverse effects were observed on any of the treated ostriches. None of the 6 treated ostriches harboured any lice, while a mean of 2446.7 immature and 12111.7 adult $S$. struthionis were recovered from the untreated ostriches. The $1 \%$ amitraz/cypermethrin pour-on was therefore $100 \%$ effective against this chewing louse.

Field efficacy study of 2 proprietary anthelmintics against infections of nematode parasites in ostriches (Struthio camelus)

\author{
J S Van der Merwe ${ }^{a}$, F J Smit ${ }^{b}$, G R Stassen ${ }^{c}$, L M Michael $^{d}$, \\ J P Louw ${ }^{\mathrm{e}}$ and A M Durand ${ }^{\mathrm{t}}$
}

${ }^{a}$ Virbac RSA, Private Bag X115, Halfway House, 1685 South Africa; ${ }^{b}$ PO Box
4511, Mokopane, 0600, South Africa: ' 42 Raubenheimer Drive, Oudtshoorn, 6620, South Africa; ${ }^{d}$ PO Box 38006, Garsfontein East, 0060, South Africa; ${ }^{2} 184$ Marija Street, Wonderboom, Pretoria, 0182, South Africa; and ${ }^{f}$ PO Box 18348, Pretoria North, 0116, South Africa; kvandermerve@virbac.co.za

Registration authorities require validation of a product in the target animal. This study was designed to evaluate the efficacy of 2 proprietary injectable ivermectin formulations against a natural infection, with artificial infection superimposed, of nematode parasites of ostriches. The artificial infection was superimposed because the ostriches purchased showed a low natural nematode load. Eighteen ostriches were involved in the trial. A single injection of a very long- (Group 1) and a long-acting (Group 2) ivermectin formulation was administered to each individual in each of the 2 treatment groups at the recommended dose rates $(0.630 \mathrm{mg} / \mathrm{kg}$ live mass for the very long-acting ivermectin and $0.2 \mathrm{mg} / \mathrm{kg}$ for the long-acting ivermectin). A control group remained untreated. Each group consisted of 6 birds. No concomitant treatment of ostriches took place. No adverse reaction to either of the 2 anthelmintics occurred in any of the treated ostriches. Only Libyostrongylus douglassi was recovered from the ostriches. The nematodes were counted and the geometric means of treated and untreated groups used to determine anthelmintic efficacy. The very long-acting ivermectin was $>99.3 \%$ effective and the long-acting ivermectin $>94.3 \%$ effective against immature and adult $L$. douglassi. Both ivermectin formulations qualify for a 'Control' anthelmintic efficacy rating. 